Original Research Article

\title{
Evaluation of cephalosporins utilization and compliance with reference to the hospital antibiotic policy of an Indian tertiary care hospital
}

\author{
Shinu Mary John ${ }^{1}$, Bijoy Kumar Panda ${ }^{1 *}$, Deepak Govind Bhosle ${ }^{2}$, Nikki Soman ${ }^{1}$
}

\begin{abstract}
${ }^{1}$ Department of Clinical
Pharmacy, Bharati Vidyapeeth, (Deemed to be University), Poona College of Pharmacy, Pune, Maharashtra, India ${ }^{2}$ Department of General Medicine, Bharati Hospital \& Research Centre, Bharati Vidyapeeth, (Deemed to be University), Pune, Maharashtra, India
\end{abstract}

Received: 02 March 2019

Revised: 23 March 2019

Accepted: 03 April 2019

*Correspondence to:

Bijoy Kumar Panda,

Email: bijoy.panda@

bharatividyapeeth.edu

Copyright: $\odot$ the author(s), publisher and licensee Medip Academy. This is an openaccess article distributed under the terms of the Creative Commons Attribution NonCommercial License, which permits unrestricted noncommercial use, distribution, and reproduction in any medium, provided the original work is properly cited.

\begin{abstract}
Background: Emergence of bacterial resistance in hospital settings due to the liberal use of antibiotics which led to an altered impact on its therapeutic efficacy and outcome, thereby increasing the treatment costs in patients. In this regard the study aims to evaluate the cephalosporins utilization and compliance to the hospital antibiotic policy in general medicine ward.

Methods: A prospective observational study was carried out over an elevenmonth period in patients prescribed with cephalosporins. Clinical data of inpatients were collected and evaluated by using World Health Organization (WHO) core prescribing indicators and defined daily dose per 100 bed-days. The comparison between the cephalosporins prescribed in the chart records to the recommendations mentioned in the institutional antibiotic policy v.2.0, help to determine the deviations in their usage pattern.

Results: A total of 370 patients were enrolled in the study, of which $54.6 \%$ were females. Cephalosporins were empirically prescribed in 240 (64.9\%) cases. The average number of total antibiotics and cephalosporins per encounter was 1.6 and 1 respectively. Among the total antibiotics, $63.7 \%$ were cephalosporins. Third generation cephalosporins $(98.4 \%)$ were commonly prescribed, with ceftriaxone (93\%) in parenteral form for respiratory tract infections (31.9\%). Total parenteral antibiotics prescribed were found to be $68.8 \%$ of which $88.6 \%$ were cephalosporins. Utilization pattern of cephalosporins amounted to be 4.95 DDD/100 bed-days. Overall compliance was achieved in 191 (51.6\%) prescriptions.

Conclusions: The rate of prescribing of cephalosporins was marginally high. There was a low rate of compliance towards policy which reflects the urgent need for repetitive intervention to comply antibiotic policy.
\end{abstract}

Keywords: Antibiotics utilization evaluation, Antibiotic policy, Compliance, Cephalosporins

\section{INTRODUCTION}

Over the decades, antibiotics have conventionally been used to treat infectious diseases in the hospital settings, which resulted in dramatic changes in the prognosis of patients with severe infections. ${ }^{1-3}$ There are trends showing an excessive or irrational use of antibiotics leading to the emergence of bacterial resistance, that had an altered impact on effective therapy and outcomes that led to increase in length of hospitalization and treatment costs in patients. ${ }^{4,5}$ Various studies demonstrated that the sales of antibiotics have increased by $40 \%$ wherein the majority of the antibiotics were cephalosporins $(60 \%){ }^{6}$

Cephalosporins are commonly used 'beta-lactam agents' which are preferred over other antibiotics due to its lower hypersensitivity reaction, broad spectrum of action, cheaper cost and better outcomes. ${ }^{7-9}$ In general, gram- 
positive microorganisms are more sensitive towards lower generation cephalosporins, while gram-negative microorganisms show more sensitivity towards higher generation cephalosporins. ${ }^{9,10}$ The availability of cephalosporins antibiotics and their various formulations along with their expanded indications have an impact on prescribing patterns in hospitals. ${ }^{5}$

In order to measure the drug use, WHO developed ATC/DDD (Anatomical Therapeutic Chemical/Defined Daily Dose) index as a specific standardized method which is periodically updated. The $D D D$ is the assumed average maintenance dose per day for a drug used for its main indication in adults. ${ }^{11}$ DDD/100 bed-days provide a rough estimate of consumption of drugs among hospital inpatients. ${ }^{11,12}$

The irrational use of antibiotic is more common in developing countries because of the societal marginalization and risk of a disease outbreak, due to unhealthy environmental conditions and inadequate nutritional intake, leading towards unauthorized and large volume sale of antibiotics. ${ }^{4}$ Despite these facts, there are rare Indian studies indicating the rational use of cephalosporins in accordance with antibiotic policy. Thus, the study was to evaluate the cephalosporins utilization and compliance with the hospital antibiotic policy in general medicine of an Indian tertiary teaching hospital. This study was undertaken to understand the quality of prescribing and compliance to antibiotic policy.

\section{METHODS}

A prospective observational drug utilization study was carried out over an eleven month period at Bharati Hospital and Research Centre, Pune, Maharashtra, India. The criteria used for the antibiotic selection incorporates antibiotic with a risk of usage abuse and high consumption rate. This study was approved by institutional ethics committee. The study population were patients admitted to the general medicine ward, of age group $\geq 18$ years, and were prescribed with cephalosporins alone or in combination with other antibiotics which was recognized manually.

A self-pre-designed form was developed for collecting the patient's details prospectively, such as demography, clinical diagnosis, lab investigation and data on prescribed cephalosporins from time to time until the patient got discharged. Cephalosporins use was classified as for definitive (based on culture reports), empirical (clinical evidence of infection without organisms being isolated) and prophylactic (without evidence of infection) therapy. The data were processed and analysed by using WHO criteria for Drug Utilization Evaluation (DUE) namely, indication, dose, route, frequency and duration of treatment as major measurements against the antibiotic policy. Additionally, the data were assessed for the WHO core drug prescribing indicators and slight modification was made to make it according to the hospital scenario before using in this study. The formula adopted from the WHO's manual includes: ${ }^{13}$

- An average number of antibiotics per encounter $=$ Number of antibiotics prescribed / Number of encounters surveyed.

- Percentage of cephalosporins within antibiotic prescribed $=$ (Number of cephalosporins prescribed / Total number of antibiotics prescribed) $\times 100$.

- An average number of parenteral antibiotics per encounter $=$ Number of parenteral prescribed / Number of encounters surveyed.

- Percentage of parenteral antibiotic prescribed = (Number of parenteral antibiotics / Total number of antibiotics prescribed) $\times 100$.

- $\quad$ Percentage of antibiotics prescribed by generic name $=($ Number of antibiotics prescribed by generic name / Total number of antibiotics prescribed) $\times 100$.

Drug utilization was also studied with the application of DDD concept and Antibiotic Consumption Index (ACI). DDD values of each cephalosporin calculated separately, the average maintenance dose for an adult weight $70 \mathrm{~kg}$ is prepared in main indications and the active substance should be taken as grams or I.U. ${ }^{11}$ The method used for inpatients is the ratio of the total DDD per 100 bed-days. This index is called ACI of the population. ${ }^{14}$

\section{$\mathrm{ACI}=\mathrm{DDD} /$ bed-days $\times 100$}

In this study, DDDs of anti-infective agents are listed for systemic use according to ATC/DDD 2016 index. ${ }^{11}$ The study was carried out for a period of 335 days; the total number of inpatients beds was 150 with occupancy index 0.82 .

The data were evaluated for the compliance of therapy with the hospital antibiotic policy (version 2.0. 2016), framed two years back which is in accordance with national treatment guidelines for antimicrobial use in infectious diseases (version 1.0.2011). ${ }^{15,16}$ The evaluation of compliance with the policy was reviewed as per the steps mentioned in Figure 1. The given therapy is said to be compliant if the prescribed antibiotic follows the right indication, dose, frequency and duration as per the policy. If there is deviation from the above-mentioned prescription pattern, then the prescribed antibiotic was considered to be non-compliant towards the policy. Data pertaining to antibiotic treatment for infectious diseases which were not mentioned in the policy or evaluation for compliance could not be performed was considered as non-assessable. Compliance was calculated as the percentage of compliant prescriptions divided by total number of prescriptions. The deviation was documented and the reason for it was ascertained from the prescriber through direct verbal access.

Data were analysed by chi-square and Fisher's exact test and expressed as mean, median, standard deviation and percentage. A $p$ value of $<0.05$ was accepted as 
statistically significant for the study. Microsoft Excel, 2010 was used for statistical data collection.

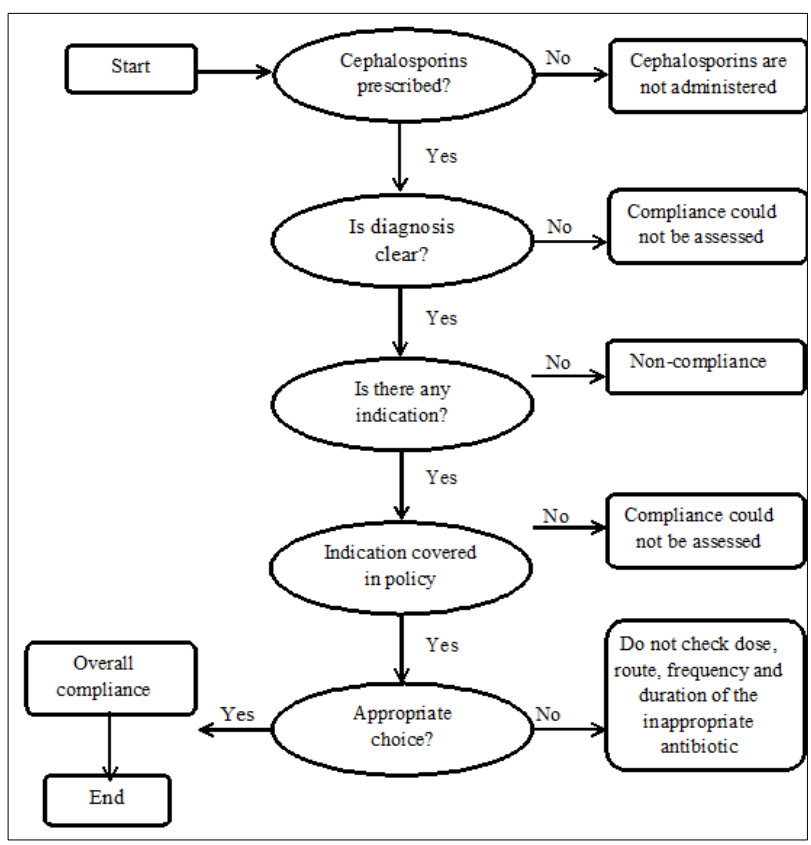

Figure 1: Steps for reviewing the records to evaluate the compliance to hospital antibiotic policy.

\section{RESULTS}

A total of 370 patients were prescribed cephalosporins, alone or in combination with other antibiotics during the study period. The demographic characteristics of patients who received cephalosporins and factors related to infection and treatment in the study population are shown in Table 1. Gender wise distribution showed that 202 $(54.6 \%)$ of patients were females. The mean age was found to be $47 \pm 19.9$ years.

Table 1: Characteristics of study population treated with cephalosporins.

\begin{tabular}{|c|c|c|}
\hline \multicolumn{3}{|c|}{ No. of patients enrolled in the study (n: 370) } \\
\hline Charact & & n $(\%)$ \\
\hline \multirow{2}{*}{ Gender } & Male & $168(45.4)$ \\
\hline & Female & $202(54.6)$ \\
\hline Age: Mean \pm SD* (Range) & Range) $\quad 47 \pm 19.9$ & (18-90 years) \\
\hline \multicolumn{2}{|c|}{ Empiric } & $240(64.9)$ \\
\hline \multicolumn{2}{|c|}{ Definitive } & $110(29.7)$ \\
\hline \multicolumn{2}{|c|}{ Prophylactic } & $20(5.4)$ \\
\hline \multicolumn{3}{|c|}{ Bacteriological investigations } \\
\hline \multicolumn{2}{|c|}{ Done } & $158(42.7)$ \\
\hline \multicolumn{2}{|c|}{ Not done } & $212(57.3)$ \\
\hline \multicolumn{3}{|c|}{$\begin{array}{l}\text { Hospitalization period of patients receiving antibiotics } \\
(\text { Mean } \pm \text { SD) }\end{array}$} \\
\hline \multicolumn{2}{|c|}{ Only one antibiotics $(n=166,44.8 \%)$} & $5.68 \pm 2.94$ \\
\hline \multicolumn{2}{|c|}{ Two antibiotics $(n=190,51.3 \%)$} & $5.73 \pm 2.89$ \\
\hline \multicolumn{2}{|c|}{ Three antibiotics $(n=14,3.8 \%)$} & $9.62 \pm 3.96$ \\
\hline
\end{tabular}

From the total cephalosporins prescribed for 370 patients, $240(64.9 \%)$ patients were administered empirically, 110 (29.7\%) for definitive purposes and $20(5.4 \%)$ patients were prescribed cephalosporins as prophylactic therapy. The median length of hospital stay was 5 days. In this study, hospitalization period of patients who received single or two antibiotics together were similar but the hospitalization period of patients receiving three antibiotics was longer (9.62 \pm 3.96$)$. Bacteriological investigations were not done in most of the patients $(57.3 \%)$ to determine the aetiology of the suspected infection. Out of the $158(42.7 \%)$ cases in which the microbial test was done, growth was observed in 44 $(27.8 \%)$ cases only.

By interpreting the data collected during the study, it was seen that the third generation cephalosporins $(98.4 \%)$ were commonly prescribed followed by the second generation. Ceftriaxone (93\%) was found to be the most commonly prescribed third-generation cephalosporins (3GCs) with a daily dose of $2 \mathrm{gm}$ in parenteral form. The mean duration of treatment with cephalosporins was 4.87 days (range 17 days). The most common indication for cephalosporins use was respiratory tract infections $(31.9 \%)$ followed by urinary tract infections (15.7\%). In 214 (57.8\%) patients co-prescribed with other antibiotics, azithromycin (45.8\%) was most commonly used.

The drug prescriptions were analysed by using WHO core indicators for usage pattern as shown in Table 2. The average number of overall antibiotics and cephalosporins per encounter was found to be 1.6 and 1 respectively. The percentage of cephalosporins prescribed in comparison to other antibiotics was found to be $63.7 \%$. The parenteral administration of overall antibiotics was found to be $68.8 \%$ of which $88.6 \%$ were cephalosporins. The total number of antibiotics prescribed in generic name was found to be $125(21.1 \%)$.

Table 2: WHO core indicators assessing the drug prescriptions.

\begin{tabular}{|lll|}
\hline Core indicators & $\begin{array}{l}\text { Total } \\
\text { antibiotics }\end{array}$ & Cephalosporins \\
\hline $\begin{array}{l}\% \text { of antibiotics } \\
\text { prescribed }\end{array}$ & $100 \%$ & $63.7 \%$ \\
\hline $\begin{array}{l}\text { Average no. of } \\
\text { parenteral antibiotics } \\
\text { per encounter }\end{array}$ & 1.09 & 1 \\
\hline $\begin{array}{l}\% \text { of parenteral } \\
\text { antibiotics prescribed }\end{array}$ & $68.8 \%$ & $88.6 \%$ \\
\hline $\begin{array}{l}\% \text { of antibiotics } \\
\text { prescribed in generic } \\
\text { name }\end{array}$ & $21.1 \%$ & 0 \\
\hline
\end{tabular}

Figure 2 shows the ACI of cephalosporin antibiotics in general medicine ward. The overall utilization pattern of cephalosporins amounted to be $4.95 \mathrm{DDD} / 100 \mathrm{bed}$-days of which, 3GCs were commonly used (4.88DDD/100 beddays). 


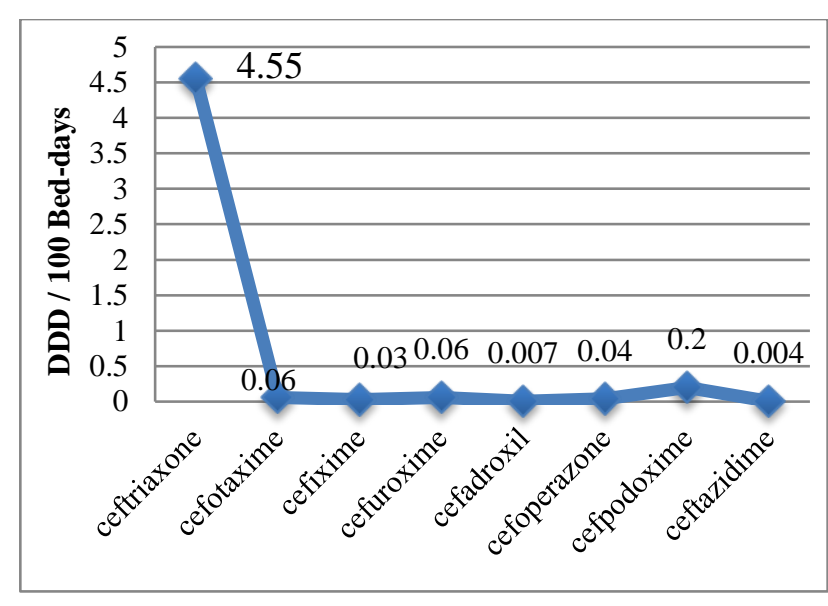

Figure 2: Antimicrobial consumption index of cephalosporins.

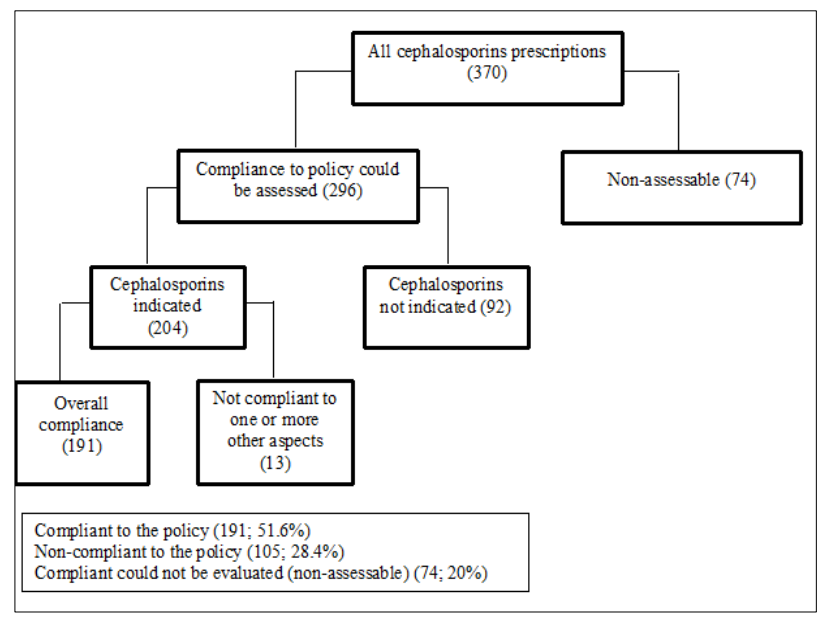

Figure 3: Situational analysis of cephalosporins prescriptions.

Table 3: Distribution of cephalosporin prescriptions by indication and level of compliance to antibiotic policy.

\begin{tabular}{|c|c|c|c|c|c|}
\hline Indication & $\begin{array}{l}\text { Number } \\
\text { n }(\%)\end{array}$ & $\begin{array}{l}\text { Compliance } \\
\text { n }(\%)\end{array}$ & $\begin{array}{l}\text { Non-Compliance } \\
\text { n }(\%)\end{array}$ & $\begin{array}{l}\mathbf{N A} \\
\mathbf{n}(\%)\end{array}$ & p value $*$ \\
\hline RTI & $118(31.9)$ & $70(59.3)$ & $8(6.8)$ & $40(33.9)$ & $<0.001$ \\
\hline UTI & $58(15.7)$ & $56(96.5)$ & $2(3.4)$ & -- & $<0.001$ \\
\hline Fever & $50(13.5)$ & $10(20)$ & $24(48)$ & $16(32)$ & 0.040 \\
\hline AGE & $46(12.4)$ & $2(4.3)$ & $44(95.7)$ & -- & $<0.001$ \\
\hline Typhoid & $36(9.7)$ & $36(100)$ & -- & -- & $<0.001$ \\
\hline Skin and Soft tissue Infection & $16(4.3)$ & $6(37.5)$ & $2(12.5)$ & $8(50)$ & -- \\
\hline CVS infection & $10(2.7)$ & $8(80)$ & -- & $2(20)$ & 0.071 \\
\hline Intra-abdominal Infections & $10(2.7)$ & $6(60)$ & $4(40)$ & -- & -- \\
\hline Meningitis & $8(2.2)$ & $6(60)$ & $2(25)$ & -- & 0.631 \\
\hline Others $^{\dagger}$ & $18(4.9)$ & $4(22.2)$ & $6(33.3)$ & $8(44.4)$ & -- \\
\hline
\end{tabular}

NA: non-assessable; RTI: respiratory tract infection; UTI: urinary tract infection; AGE: acute gastroenteritis

$\uparrow$ Viral infections, Dengue, Rickettsial fever

$*$ p Value (excluding non-assessable) - Chi square test and Fishers exact test

Table 4: Distribution of cephalosporins and level of compliance based on DUE* criteria. $\dagger$

\begin{tabular}{|lll|}
\hline DUE criteria & $\begin{array}{l}\text { Compliance } \\
\text { n(\%) }\end{array}$ & $\begin{array}{l}\text { Non-compliance } \\
\text { n(\%) }\end{array}$ \\
\hline Indication & 204(55.1\%) & $92(24.9 \%)$ \\
\hline Dose & $204(55.1 \%)$ & $92(24.9 \%)$ \\
\hline Frequency & $204(55.1 \%)$ & $92(24.9 \%)$ \\
\hline Duration & $191(51.6 \%)$ & $105(28.4 \%)$ \\
\hline
\end{tabular}

*DUE: Drug Utilization Evaluation

$\dagger$ Excluding non-assessable cases

The situational analysis of all cephalosporin prescriptions is interpreted in Figure 3. The compliance of cephalosporins administration according to the antibiotic policy (version 2.0. 2016) was evaluated for indication, dose, frequency and duration of administration in a total of 370 patients. ${ }^{15}$ Among the total 191 (51.6\%) were compliant, whereas $105(28.4 \%)$ cases were noncompliant and the rest $74(20 \%)$ of the data was nonassessable.

Table 3 and 4 shows the distribution of cephalosporin prescriptions by DUE criteria and level of compliance of therapy with the antibiotic policy. From the total of 105 (28.4\%) non-compliant cases, $92 \quad(87.6 \%)$ elicited differences in indication, duration, dose and frequency, whereas, $13(12.4 \%)$ shows non-compliance only in duration of therapy as per the policy.

\section{DISCUSSION}

Cephalosporins are the most widely used class of antibiotics that need careful monitoring to ensure their rationale use in this era where there is an increased threat due to microbial resistance. ${ }^{5}$ The present study shows the 
marginally higher utilization of cephalosporins in general medicine $(63.7 \%)$ which was similar to the studies reported by Gururaja et al, and Reddy et al, where most of the study patients belonged to medicine department. ${ }^{5,7}$ Gender wise distribution of inpatients shows that cephalosporin use in female $(54.6 \%)$ was more than male $(45.4 \%)$ population. This may be due to the fact that women are more susceptible towards bacterial infections, especially Urinary Tract Infections (UTIs), and Respiratory Tract Infections (RTIs). This is similar to the results obtained in Sri Ramachandra Hospital, Chennai which shows a female predominance $(61.8 \%)$ over male $(38.2 \%)$ population. ${ }^{17}$ Whereas, various studies have reported that cephalosporin use in the male population is more as compared to female..$^{7,18-20}$

The present study revealed that 240 (64.9\%) patients received cephalosporins as empirical prescriptions. This is because the microbiological results cannot be availed within $24-72 \mathrm{hrs}$. The initial therapy is started on the basis of physician's clinical judgment, patient's clinical condition, and laboratory parameters. This is similar to the studies conducted at a University hospital in West Indies, where two-thirds of the patients $(67.9 \%)$ were treated with empiric antibiotics and Babu et al, where $74.26 \%$ was of empiric therapy. ${ }^{21,22}$ In this study, it was observed that $29.7 \%$ of patients were prescribed cephalosporins on the basis of culture results and confirmatory diagnostic tests. In many cases, no microbial growth was detected; this may be the reason for the low percentage usage of cephalosporins for the definitive therapy.

Bacteriological investigation was done only on 158 $(42.7 \%)$ patients who had severe infection or shows unresponsiveness to therapy. Whereas in other patients investigations were usually not suggested due to nonaffordability, prior initiation of antibiotic therapy and delay in attaining results. The findings were similar to the studies conducted at the Tikur Anbessa specialized hospital and university hospital of West Indies. ${ }^{21,23}$

Certain factors such as drug or host-related response pertaining to antibiotic therapy may lead to the failure of treatment. The number of antibiotics use may lead to an increment in treatment cost incurring a financial loss due to increased length of stay in hospitalized patients. ${ }^{4}$ In this study, the hospitalization period of a patient who received three antibiotics was high $(\mathrm{P}=0.003)$; this may increase the hospital infection risk and treatment cost. This was similar to the study conducted in Turkey where the use of three antibiotics was inappropriate and the incidence of hospital stay was twice in patients. ${ }^{4}$

The study reveals that the 3 GCs $(98.4 \%)$ were most commonly prescribed, followed by second generation $(1.1 \%)$. This result is in agreement with the results of various other studies that were reported.,7,24 Another similar study conducted by Jonathan et al shows higher usage of 3GCs $(95.85 \%)$, whereas a study conducted in a teaching hospital in Nepal shows a low use of 3GCs. ${ }^{18,25}$
This higher use may be due to its broad spectrum of activity against most of the bacterial species, responsible for causing infections, especially against gram-negative microorganisms as well as the routine availability of these drugs in this setup. They have been successfully used in controlling and treating infections. Their rapid use in hospital settings to treat various infections has increased, exerting a significant influence over the rates of multidrug-resistant nosocomial pathogens. ${ }^{26}$ It was observed that ceftriaxone $(93 \%)$ was the most commonly prescribed cephalosporins, due to its high anti-bacterial potency, wide spectrum of activity and low potency of toxicity. ${ }^{8,9,23}$ Similar results were obtained from various studies. ${ }^{7,19,24,27,28}$ But it was quite different from the results of a study conducted by Kaliamoorthy et al, in a tertiary care hospital, in which ceftriaxone was ranked third in terms of utilization rate $(19.5 \%)$ following cefixime $(32.7 \%)$ and cefotaxime $(31.3 \%){ }^{17}$ The use of cephalosporins was found to be high for the treatment of RTIs. The findings were similar to results obtained from various studies..$^{8,23-25,27-28}$

The present study reveals that the mean duration of treatment with cephalosporins in the hospital was 4.87 days. The duration of antibiotics depends on the severity of the infections. Mild-moderate conditions such as UTI, typhoid, RTIs required 1-7 days of treatment duration. This signifies that the treatment duration of cephalosporins in such conditions was in accordance with the antibiotic policy. This finding was similar to the results obtained from the study conducted by Reddy et al, where the duration was 5 days. $^{7}$

It was observed that an average number of overall antibiotics and cephalosporins prescribed per encounter were found to be 1.6 and 1 respectively. The percentage of cephalosporins prescribed $(63.7 \%)$ compared to other antibiotics, was higher than the standard (20.0\%-26.8\%) derived to be ideal. ${ }^{13}$ This may promote bacterial dissemination and resistance which suggests a need for antibiotic regulation in cephalosporin prescribing pattern. ${ }^{29}$ A high percentile of antibiotics prescribed in this study may be due to patient's expectation to receive antibiotics or the prescriber's belief that the therapeutic efficacy of antibiotic is low. Parenteral antibiotics prescribed were found to be $68.8 \%$ of which $88.6 \%$ was cephalosporins. The results of this study were high as compared to the standards recommended by WHO $(13.4 \%-24.1 \%) .{ }^{13}$ In this hospital, parenteral cephalosporins are mostly preferred according to the antibiotic policy. Once the antibiotics administered to patients were switched from parenteral to oral therapy, the patients recovered faster and were unwilling to stay any further. Myths amongst patients regarding faster efficacy and recovery due to parenterally administered antibiotics when compared to its oral form prompt the physician to administer parenteral antibiotics. This will increase the cost as well as the risk of transmission of potentially serious diseases through the unhygienic use of injectables. Antibiotics were prescribed in their brand names instead 
of generic names. This value is less as compared with the standards of WHO $(100 \%){ }^{13-29}$

The use of cephalosporins has widely been assessed in the hospital by measuring quantitative pharmacy data to calculate the number of DDD per number of bed-days. ${ }^{11}$ The ACI of cephalosporins was 4.95 DDD/100 bed-days of which, 3GCs were commonly used (4.88DDD/100 beddays). The use of first and second generation cephalosporins was low, and that of the 3GCs was higher than that reported in Nepal study. ${ }^{18}$ The DDD values were slightly higher than those previously reported from the medicine ward of the Manipal Teaching hospital. ${ }^{19}$ Ceftriaxone was the most commonly consumed (4.55 DDD/100 bed-days) parenteral cephalosporin and its frequent use may lead to drug resistance.

The major goal and challenge of antibiotic policies is the proper assessment and evaluation of patient's disease condition and rational prescribing pattern of antibiotics by the physician, and proper medication adherence by the patients. This approach would help to prevent antibioticmicrobial resistance, diminish cost, improves quality of patient life and antibiotic usage. ${ }^{30}$ In this study, 191 of the 370 patient's charts $(51.6 \%)$ were found to be complying for an overall evaluation of indication, dose, and frequency and duration of treatment according to the antibiotic policy. The majority (28.4\%) of the non-compliance to the policy were related to differences in indication, dose, route, frequency, and duration of administration between practice and the policy. Wrong uses of cephalosporins in the assessments of acute gastroenteritis (AGE), rickettsial fever, complicated UTI, viral conditions, Chronic Obstructive Pulmonary Disease (COPD), and Bronchitis for which the policy recommends no indication of the drug was noticed in the study. Co-trimoxazole or piperacillintazobactam (blood in stool or culture) is recommended, instead of ceftriaxone for the management of AGE as per the policy. In a similar manner, the choice of antibiotic for complicated UTI is meropenem whereas for rickettsial fever is doxycycline or azithromycin. The possible reasons for the non-compliance are the consideration of antibiotics for speedy recovery leading to the lack of awareness and ignorance, exerting pressure on physicians to empirically prescribed antibiotics. This may prompt the rise of the bacterial resistance which in turn compromises antibiotic effectiveness leading to treatment failure, increased treatment cost as well as risk to the patient safety. Limitations of the study include inadequacy of information given by physicians regarding deviations from policy, inaccurate or missing data's from patient charts and insufficient data capturing due to prospective study type.

\section{CONCLUSION}

The increased use of cephalosporins in the hospital settings is a matter of concern. Our study highlights the trend of marginally higher utilisation of cephalosporins especially 3GCs. A significant number of patients received cephalosporins, which was not in accordance with the hospital antibiotic policy. The recommendations that could improve the compliance are quickening the availability of culture and sensitivity reports, accessibility of guidelines on the hospital information system, repetitive audits to monitor antibiotic misuse and update physician knowledge regarding rational prescribing. Involvement of a clinical pharmacist, in order to enhance compliance and rational antibiotic therapy may improve the quality of patient care.

\section{ACKNOWLEDGEMENTS}

Authors would like to thank to the college staff members for unreserved guidance and timely support. Authors express their thanks to the Department of Medicine, Bharati Hospital and Research Centre, Pune, for helping them in the completion of the study.

\section{Funding: No funding sources}

Conflict of interest: None declared

Ethical approval: The study was approved by the Institutional Ethics Committee of Bharati Vidyapeeth (Deemed to be University) Medical College, Pune, Maharashtra, India (Ref: BVDU/MC/E6; 1/09/2016)

\section{REFERENCES}

1. Thu T, Rahman M, Coffin S, Harun-Or-Rashid M, Sakamoto J, Hung N. Antibiotic use in Vietnamese hospitals: A multicenter point-prevalence study. Am J Infect Control. 2012;40(9):840-4.

2. Hulscher ME, Grol RP, van der Meer JW. Antibiotic prescribing in hospitals: a social and behavioral scientific approach. Lancet Infect Dis. 2010;10:16775.

3. Finch R. Antimicrobial therapy: principles of use. Medicine. 2005;33:42-6.

4. Tünger Ö, Dinç G, Özbakkaloglu B, Atman Ü, Algün Ü. Evaluation of rational antibiotic use. Int $\mathrm{J}$ Antimicrob Agents. 2000;15(2):131-5.

5. Gururaja MP, Sarah A, Samaga L, Joshi H, Nair S, Shastry CS. Cephalosporin utilization evaluation in a university teaching hospital: prospective study. J Drug Deliv Therapeut. 2013;3(2):83-7.

6. Ganguly NK, Arora NK, Chandy SJ, Fairoze MN, Gill JP, Gupta U, et al. Rationalizing antibiotic use to limit antibiotic resistance in India. Indian $\mathrm{J}$ Med Res. 2011;134:281-94.

7. Reddy ES, Reza DH, Sankar B, Mubarak A, Ahamadasafnamariyam. Drug utilization evaluation of cephalosporins in the general medicine and general surgery departments in a Tertiary care teaching hospital. Am J Pharm Health Res. 2015;3(6):23213647.

8. Ayinalem G, Gelaw B, Belay A, Linjesa J. Drug use evaluation of ceftriaxone in medical ward of Dessie Referral Hospital, North East Ethiopia. Int J Chem Natur Sci. 2013;1(1):12-6.

9. Abebe FA, Derbew F, Berhe DF, Berhe AH, Hishe HZ, Akaleweld MA. Drug use evaluation of 
Ceftriaxone: the case of Ayder Referral Hospital, Mekelle, Ethiopia. IJPSR. 2012;3(7):2191-5.

10. Christopher J, Denis B. Cephalosporins: A review. Pediatrics in review. 2008;29(8):264-73.

11. WHO Collaborating Centre for Drug Statistics Methodology: Guidelines for ATC classification and DDD assignment Oslo; 2016.

12. Gordana P, Zorica J, Kavin V. Application of the ATC/DDD methodology to compare antibiotic utilization in two university hospital surgical departments. Med Biol. 2005;12(3):174-8.

13. Vooss AT, Diefenthaeler HS. Evaluation of prescription indicators established by the WHO in Getulio Vargas - RS. Braz J Pham Sci. 2011;47(2):385-90.

14. Sözen H, GönenI, Sözen, A, Kutlucan A, Kalemci S, Sahan M. Application of ATC/DDD methodology to evaluate of antibiotic use in a general hospital in turkey. Ann Clin Microbiol Antimicrob. 2013;12(1):23.

15. Hospital Infection Control Committee, Antibiotic Policy, version 2.0, 1.11.2016. Pune: Bharati Vidyapeeth (Deemed to be University) Medical College Hospital and Research Center; 2016:1-63.

16. Venkatesh S, Chauhan LS, Gadpayle AK, Jain, TS, Ghafur A, Wattal C et al., National Treatment Guidelines for Antimicrobial Use in Infectious Diseases. India: National Centre For Disease Control, MOHFW, Government of India; 20111:1-64.

17. Kaliamoorthy K, Sankaralinjam R, punniyakotti S, Janardhan V, Cheekala UMR. Drug utilization evaluation of third generation cephalosporins using core drug use indicators. Pak J Pharm Sci. 2012;25(2):339-42.

18. Shankar P, Subish P, Upadhyay D, Dubey A, Deshpande V. Cephalosporin utilization in the inpatient wards of a teaching hospital in Western Nepal. JIOM. 2005;27:7-12.

19. Shankar RP, Partha P, Shenoy NK, Easow JM, Brahmadathan KN. Prescribing patterns of antibiotics and sensitivity patterns of common microorganisms in the Internal Medicine ward of a teaching hospital in western Nepal: a prospective study. Ann Clin Microbiol Antimicrob. 2003;2:7.

20. Arul B, Rangabashyam SR, Kothai R, Bobby MT, Elavarasi M, Glory MA. Drug utilization review of cephalosporins in a tertiary care hospital: a retrospective study. World J Pharm Pharm Sci. 2017;6(9):1737-43.

21. Chin V, Harding HE, Tennant I, Soogrim D, GordonStrachan GM, Frankson MA. Dynamics of antibiotic usage in the intensive care unit at the University hospital of the West Indies. West Indian Med J. 2010;59(2):159-64.

22. Babu J, Jyothi K. Drug utilization evaluation of cephalosporins in general medicine units of rural tertiary care hospital. Int $\mathrm{J}$ Curr Pharm Res. 2012;4(2):88-91

23. Sileshi A, Tenna A, Feyissa M, Shibeshi W. Evaluation of ceftriaxone utilization in medical and emergency wards of Tikur Anbessa specialized hospital: a prospective cross sectional study. BMC Pharmacol Toxico. 2016;17:7.

24. Abou-Shaaban M, Ali A, Rao P, Majid A. Drug utilization review of cephalosporins in a secondary care hospital in United Arab Emirates. Int $\mathrm{J}$ Clin Pharm. 2016;38(6):1367-71.

25. Marion B, Tony MK, Jonathan GA. Ceftriaxone and cefotaxime use in Victorian hospitals. Med J Aust. 2002;176(11):524-9.

26. Kheder SI. Cephalosporin Usage and Resistance Trend in a Sudanese Hospital Surgical Wards. JPBMS. 2011;11(11).

27. Pinto Pereira L, Phillips M, Ramlal H, Teemul K, Prabhakar P. Third generation cephalosporin use in a tertiary hospital in Port of Spain, Trinidad: need for an antibiotic policy. BMC Infect Dis. 2004;4(1):59.

28. Ayele AA, Gebresillassie BM, Erku DA, Gebreyohannes E, Demssie DG, Mersha AG, et al. Prospective evaluation of Ceftriaxone use in medical and emergency wards of Gondar university referral hospital, Ethiopia. Pharmacol Res Perspect. 2018;6(1):e00383.

29. Isah AO, Ross-Degnan D, Quick J, Laing R, Mabadeje AFB. The development of standard values for the WHO drug use prescribing indicators. ICUM/EDM/WHO. Available at: http://www.archives.who.int/prduc2004/rducd/ICIU M_Posters/1a2_txt.html. Accessed 16 Aug 2016.

30. Nathwani D. How do you measure the impact of an antibiotic policy? J Hosp Infect. 1999;43(Suppl):2658 .

Cite this article as: Shinu MJ, Panda BK, Bhosle DG, Soman N. Evaluation of cephalosporins utilization and compliance with reference to the hospital antibiotic policy of an Indian tertiary care hospital. Int J Basic Clin Pharmacol 2019;8:1044-50. 